\title{
BERGMAN KERNELS ON RESOLUTIONS OF ISOLATED SINGULARITIES
}

\author{
Hing-Sun LuK ${ }^{\dagger}$, Stephen S.T. Yau*i , And Larn-Ying YeH \\ In memory of S. Bergman.
}

\section{Introduction}

The Bergman kernel is a basic biholomorphic invariant on domains in $\mathbb{C}^{n}$. A lot of important work has been done on its explicit computation and asymptotic expansion. Generalized to complex manifolds [K], the Bergman kernel continues to play a fundamental role. However, it seems that there is little attention given to the possible role of the Bergman kernel on analytic spaces, in connection with the study of singularities.

In this paper, we give an initial step in studying the Bergman kernel on a resolution of an isolated 2-dimensional singularity. The problem is to investigate how the Bergman kernel holds information on the singularity. Our main result (Theorem 5 and Theorem 7) is that for any Gorenstein surface singularity, the exceptional set of the resolution is exactly the minimal set of the Bergman kernel. The rational singularities will be studied explicitly and a canonical holomorphic 2 -form will be given. Since the exceptional set provides crucial topological information on the resolution, our result shows that the analytic definition of the Bergman kernel contains important topological information on the singularity.

We wish to thank the referees for useful comments which help us in preparing the revised version. The second author would like to express his gratitude to the Late Professor Bergman who suggested the topic to him twenty-six years ago when he was a graduate student working on singularities.

\section{Bergman kernel and monoidal transformation}

Let $M$ be a complex $n$-dimensional manifold. We recall the definition of the Bergman kernel. Let $F$ be the set of all holomorphic $n$-forms $\varphi$ on $M$ such that $\left|\int_{M} \varphi \wedge \bar{\varphi}\right|<\infty$. ( $\varphi$ will be called $L^{2}$ or square integrable.) $F$ is a separable complex Hilbert space under the inner product $\left(\varphi_{1}, \varphi_{2}\right)=(\sqrt{-1})^{n^{2}} \int_{M} \varphi_{1} \wedge$ $\bar{\varphi}_{2}$. The corresponding norm $(\varphi, \varphi)^{\frac{1}{2}}$ will be denoted by $\|\varphi\|$. Let $\left\{\omega_{j}\right\}$ be a complete orthonormal basis of $F$. Then $K(z, \bar{w})=\sum \omega_{j}(z) \wedge \overline{\omega_{j}(w)}$ can be

Received January 26, 2000.

$\dagger$ Research partially supported by Hong Kong RGC.

* Research partially supported by NSF. 
shown to converge uniformly on compact subsets to a holomorphic $2 n$-form on $M \times \bar{M}$. Here, $\bar{M}$ denotes the conjugate complex manifold obtained by taking the conjugate coordinate charts of $M$. Further, $K(z, \bar{w})$ is independent of the choice of complete orthonormal basis of $F$. If each point $z \in M$ is identified with the point $(z, \bar{z}) \in M \times \bar{M}$, then $K(z)=K(z, \bar{z})$ can be regarded as a $2 n$-form on $M . K(z)$ is referred to as the Bergman kernel of $M$. Since the Hilbert space $F$ with its inner product is invariant under biholomorphic maps, so is the Bergman kernel.

Theorem 1. Let $M$ be a complex n-dimensional manifold and $A$ be a compact submanifold of $M$. Let $\pi: M_{1} \rightarrow M$ be the blow up of $M$ along $A$. Assume that $K_{M}(z)$ is well-defined. Then we have $K_{M_{1}}(z)=\pi^{*} K_{M}(z)$.

Proof. Let $F, F_{1}$ be the respective spaces of $L^{2}$ holomorphic $n$-forms on $M, M_{1}$. Since $\pi^{*}$ pulls back $L^{2}$ holomorphic $n$-forms on $M$ to $L^{2}$ holomorphic $n$-forms on $M_{1}$, preserving inner product, we have an injective map $\pi^{*}: F \rightarrow F_{1}$.

Consider any $\varphi_{1} \in F_{1} . \varphi_{1}$ defines a $L^{2}$ holomorphic $n$-form $\varphi$ on $M \backslash A$. Then $\varphi$ extends to a holomorphic $n$-form on the complex manifold $M$. Clearly $\varphi \in F$ and $\pi^{*} \varphi=\varphi_{1}$. Hence $\pi^{*}: F \rightarrow F_{1}$ is surjective.

For any complete orthonormal basis $\left\{\omega_{j}\right\}$ of $F,\left\{\pi^{*} \omega_{j}\right\}$ is a complete orthonormal basis of $F_{1}$. Hence

$$
K_{M_{1}}=\sum \pi^{*} \omega_{j} \wedge \overline{\pi^{*} \omega_{j}}=\pi^{*} \sum_{j} \omega_{j} \wedge \overline{\omega_{j}}=\pi^{*} K_{M}
$$

We propose to study the Bergman kernel on a resolution of an isolated 2dimensional singularity. More precisely, let $(\widetilde{V}, 0)$ be a normal surface singularity in $\mathbb{C}^{n}$ and $\widetilde{\pi}: \widetilde{M} \rightarrow \widetilde{V}$ be a resolution of $\widetilde{V}$. We choose an open ball $B$ in $\mathbb{C}^{n}$ centered at 0 such that 0 is the only singularity in the closure of $V=\widetilde{V} \cap B$. We shall consider $M=\widetilde{\pi}^{-1}(V)$ and denote by $\pi: M \rightarrow V$ the restriction of $\tilde{\pi}$ to $M$. It is known that for a normal surface singularity, every holomorphic function in a deleted neighborhood of the singularity extends through the singularity.

Resolutions of isolated singularities are not unique. However, it is well known [L1, Theorem 5.10] that for any two resolutions $\pi_{i}: M_{i} \rightarrow V, i=1,2$, of a normal 2-dimensional singularity, there exist a resolution $\pi: M \rightarrow V$ and factorization $\theta_{i}: M \rightarrow M_{i}$ such that $\pi=\pi_{i} \theta_{i}, i=1,2$, and $\theta_{1}, \theta_{2}$ are iterated blow ups. Hence, according to Theorem 1, the choice of resolution is not important for the study of the Bergman kernel.

In what follows, we shall assume that $(V, 0)$ is a Gorenstein surface singularity. This means that there exists a holomorphic 2 -form $\varphi_{0}$ on $V \backslash\{0\}$ which is nowhere vanishing on $V \backslash\{0\}$, i.e. the canonical bundle on $V \backslash\{0\}$ is trivial. We remark that hypersurface and complete intersection singularities are Gorenstein singularities. Further, we shall work, in the remainder of the paper, with the minimal resolution $\pi: M \rightarrow V$. 


\section{The minimal set of the Bergman kernel}

Let $E=\pi^{-1}(0)$ be the exceptional set in $M$. Let $\varphi_{0}$ be a 2 -form on $V \backslash\{0\}$ as described above. We shall denote the pull back $\pi^{*}\left(\varphi_{0}\right)$ also by $\varphi_{0}$. The context will be made clear so that no confusion would arise.

The geometric genus of $V$ is given by

$$
p_{g}=\operatorname{dim} H^{1}(M, \mathcal{O})=\operatorname{dim} \Gamma\left(M \backslash E, \Omega^{2}\right) / \Gamma\left(M, \Omega^{2}\right)
$$

For a reference to the identification of $H^{1}(M, \mathcal{O})$ with the quotient space of global sections, see [Y, p.56]. 0 is defined to be a rational singularity if $p_{g}=0$.

Proposition 2. Under the conditions given above, $p_{g}=0$ if and only if $\varphi_{0}$ is a holomorphic 2-form on $M$.

Proof. Let $\psi$ be any element of $\Gamma\left(M \backslash E, \Omega^{2}\right)$. $\psi$ defines a holomorphic 2-form on $V \backslash\{0\}$, which we also denote by $\psi$. On $V \backslash\{0\}, \psi=h \varphi_{0}$ for some holomorphic function $h$. As noted above, $h$ extends to a holomorphic function on $V$. If we compose $\pi$ and the extension of $h$, then on $M \backslash E$, we have $\psi=h \varphi_{0}$, where $h$ is now a holomorphic function on $M$. Thus, if $\varphi_{0}$ is holomorphic on $M$, then so is $\psi$, which implies that $p_{g}=0$. Conversely, if $\varphi_{0}$ is not holomorphic on $M$, then $\varphi_{0} \in \Gamma\left(M \backslash E, \Omega^{2}\right) \backslash \Gamma\left(M, \Omega^{2}\right)$, hence $p_{g} \geq 1$.

Our situation is clarified by the following theorem of Laufer.

Theorem 3. (Laufer [L2], see also [Y]) A normal surface singularity is rational if and only if any holomorphic 2-form on a deleted neighborhood of the singular point is square integrable.

On the other hand, we have the following lemma.

Lemma 4. Let $(V, 0)$ be a normal surface singularity and $M$ be the minimal resolution of $V$. Let $E$ be the exceptional set of $M$. Let $\eta$ be a meromorphic 2-form on $M$ which is holomorphic and nowhere zero on $M \backslash E$. If $\eta$ is not holomorphic on $M$, then $E$ is the pole set of $\eta$.

Proof. Let $E=\cup_{i=1}^{n} A_{i}$ be the irreducible decomposition of $E$. Then $(\eta)=$ $-\sum_{i=1}^{k_{1}} a_{i} A_{i}+\sum_{i=k_{2}+1}^{n} b_{i} A_{i}$, where $a_{i}>0, b_{i}>0$ and $k_{2} \geq k_{1} \geq 1$. Let $K$ be the canonical divisor on $M$. Then $K=(\eta)$. Since $M$ is the minimal resolution, we have by the adjunction formula, $A_{j} \cdot K \geq 0$ for all $A_{j} \subset E$, i.e.

$$
A_{j} \cdot\left(\sum_{i=1}^{k_{1}} a_{i} A_{i}-\sum_{i=k_{2}+1}^{n} b_{i} A_{i}\right) \leq 0, \text { for all } j .
$$

We want to prove that $k_{1}=n$. Suppose on the contrary that $k_{1}<n$. Recall that by a theorem of Artin [A] [L2], there exists a unique minimal positive cycle $Z=\sum z_{i} A_{i}$, where all $z_{i}>0$, with the property that $A_{j} \cdot Z \leq 0$ for all $A_{j} \subset E$. 
Let $Y=\sum_{i=1}^{k_{1}} a_{i} A_{i}-\sum_{i=k_{2}+1}^{n} b_{i} A_{i}$. Then $A_{j} \cdot Y \leq 0$ for all $A_{j} \subset E$. Since $k_{1}<n$, we can create an effective cycle $Y_{1} \neq 0$ with the following properties: $A_{j} \cdot Y_{1} \leq 0$ for all $A_{j} \subset E$ and for some $A_{i_{0}} \subset E$, the coefficient of $A_{i_{0}}$ in $Y_{1}$ is zero. In fact, if $k_{2}=n$ and there is no $b_{i}$, let $Y_{1}=Y$. If $k_{2}<n$, by taking positive linear combination of $Y$ and $Z$ (i.e. $c_{1} Y+c_{2} Z, c_{1}, c_{2}>0$ ), we can reduce the number of negative coefficients in $Y$ by at least one and get at least one zero coefficient. Repeating the procedure if necessary, we get $Y_{1}$.

$Y_{1}$ contradicts the minimality of $Z$, as follows. Let $Y_{1}=\sum y_{1 i} A_{i}$ and consider $Y_{2}=\sum \min \left(y_{1 i}, z_{i}\right) A_{i}$ (i.e. $\left.Y_{2}=\min \left(Y_{1}, Z\right)\right)$. Since $z_{i_{0}}>0$ and $y_{1 i_{0}}=0$, $Z \not \leq Y_{2}$. To see that $A_{j} \cdot Y_{2} \leq 0$ for any fixed $A_{j} \subset E$, observe that since $A_{j} \cdot A_{i} \geq 0$ for all $i \neq j, A_{j} \cdot Y_{2} \leq A_{j} \cdot Y_{1}$ (respectively $A_{j} \cdot Z$ ) if $\min \left(y_{1 j}, z_{j}\right)=y_{1 j}$ (respectively $z_{j}$ ).

It is now natural to divide our discussion into two cases : Case I $p_{g} \geq 1$ and Case II $p_{g}=0$. In each case, we shall introduce an invariant notion of minimal set for the Bergman kernel on $M$.

Case I. When $p_{g} \geq 1$, pick any meromorphic 2-form $\eta$ on $M$ satisfying the following conditions:

(1) $\eta$ is holomorphic and nowhere zero on $M \backslash E$.

(2) $E$ is the pole set of $\eta$.

By Proposition 2 and Lemma 4, one such choice of $\eta$ is $\varphi_{0}$. (Since $\varphi_{0}$ is not canonical, we consider all choices of $\eta$.)

Observe that since $V=\widetilde{V} \cap B$ has no singularities in its closure other than 0 , (1) implies that $\eta$ is $L^{2}$ on the complement of a neighborhood of $E$ in $M$.

Theorem 5. Under the above conditions of Case $I, \frac{K(z, \bar{z})}{\eta(z) \wedge \overline{\eta(z)}}$ defines a nonnegative real valued function $\sigma$ on $M$. The zero set of $\sigma$ is exactly the exceptional set $E$. We shall refer to the zero set of $\sigma$ as the minimal set of the Bergman kernel on $M$ in this case.

Proof. Let $\left\{\omega_{j}\right\}$ be a complete orthonormal basis of the space $F$ of all $L^{2}$ holomorphic 2-forms on $M$. As in the proof of Proposition 2, each $\omega_{j}=h_{j} \eta$ for some holomorphic function $h_{j}$ on $M$ which vanishes on $E$. Then $K(z, \bar{z})=\sum_{j} \omega_{j}(z) \wedge$ $\overline{\omega_{j}(z)}=\left(\sum_{j}\left|h_{j}(z)\right|^{2}\right) \eta(z) \wedge \overline{\eta(z)}$. Hence, $\sigma(z)=\frac{K(z, \bar{z})}{\eta(z) \wedge \overline{\eta(z)}}=\sum_{j}\left|h_{j}(z)\right|^{2} \geq 0$ and $\sigma=0$ on $E$.

Observe that there exists $k \in \mathbb{N}$ such that each $z_{1}^{k} \eta, \ldots, z_{n}^{k} \eta\left(z_{1}, \ldots, z_{n}\right.$ being the coordinates of $\left.\mathbb{C}^{n}\right)$ is a holomorphic 2 -form on $M$, by virtue of the finiteness of $p_{g}$. Since $\eta$ is $L^{2}$ on the complement of a neighborhood of $E, z_{1}^{k} \eta, \ldots, z_{n}^{k} \eta$ are $L^{2}$ on $M$ hence belong to $F$. Applying the Gram-Schmidt process to these elements of $F$, we get orthonormal elements $g_{i} \eta$ with the same linear span in $F$, 
for some holomorphic functions $g_{i}$ on $M$. Then we can extend $\left\{g_{i} \eta\right\}$ to a complete orthonormal basis of $F$, by means of which, we see that $\sigma=\frac{K}{\eta \wedge \bar{\eta}} \geq \sum_{i}\left|g_{i}\right|^{2}$.

Now for any $p \in M \backslash E, \pi(p) \neq 0$. Not all $z_{1}, \ldots, z_{n}$ are zero at $\pi(p)$, hence some $g_{i}(p) \neq 0$ and $\sigma(p)>0$.

Case II. When $p_{g}=0,0$ is a rational singularity. First, note that a Gorenstein rational singularity is actually a rational double point. This can be seen as follows. Since $(V, 0)$ is a Gorenstein rational singularity, the canonical divisor $K$ in $M$ is effective with support in $E\left(=\cup_{i} A_{i}\right)$, i.e. $K=\sum n_{i} A_{i}, n_{i} \geq 0$. By the adjunction formula, $A_{i} \cdot K \geq 0$ for all $A_{i} \subset E$. We claim that $K=0$. Suppose on the contrary that $K=\sum_{i=k}^{n} n_{i} A_{i}$, where $n_{i}>0$ and $k \leq n$. Then $-K=\sum_{i=k}^{n}\left(-n_{i}\right) A_{i}$ and $A_{i} \cdot(-K) \leq 0$ for all $A_{i}$. By the same argument as in the proof of Lemma 4, we get a contradiction to the minimality of $Z$. Hence $K=0$ and $A_{i} \cdot K=0$ for all $A_{i}$. The adjunction formula also gives $A_{i}^{2}=-2$ for all $A_{i}$. Then it can be shown that the weighted dual graph of $E$ is one of those of rational double points. By the tautness of rational double points, we are done.

In particular, $(V, 0)$ is a hypersurface singularity given by a defining equation $f(x, y, z)=0 . f$ provides us with a useful 2-form as follows. Let $\iota: V \rightarrow \mathbb{C}^{3}$ be the inclusion map. Since $\iota^{*}(d f)=0$, we have

$$
\iota^{*}\left(\frac{d y \wedge d z}{f_{x}}\right)=\iota^{*}\left(\frac{d z \wedge d x}{f_{y}}\right)=\iota^{*}\left(\frac{d x \wedge d y}{f_{z}}\right),
$$

which defines a meromorphic 2 -form $\varphi_{0}$ on $V$. Since $f_{x}, f_{y}, f_{z}$ vanish simultaneously only at $0 \in V, \varphi_{0}$ is holomorphic and nowhere zero on $V \backslash\{0\}$. Hence, the pull back $\pi^{*}\left(\varphi_{0}\right)$ is a meromorphic 2-form on $M$, which is holomorphic and nowhere zero on $M \backslash E$. For simplicity, we shall write

$$
\varphi_{0}=\frac{d y \wedge d z}{f_{x}}=\frac{d z \wedge d x}{f_{y}}=\frac{d x \wedge d y}{f_{z}}
$$

and denote the pull back $\pi^{*}\left(\varphi_{0}\right)$ also by $\varphi_{0}$. It turns out further that $\varphi_{0}$ is an $L^{2}$ nowhere vanishing holomorphic 2 -form on $M$; this will be verified explicitly in Section 3.

Consider the subspace $F^{\prime}=\{\varphi \in F: \varphi$ vanishes on $E\}$.

Proposition 6. Under the above conditions of Case $I I, \operatorname{dim} F / F^{\prime}=1$.

Proof. Consider any $\varphi \in F$. Since $\varphi_{0}$ is nowhere zero on $M, \frac{\varphi}{\varphi_{0}}=h$ for some holomorphic function on $M . h$ is constant on $E$, say equal to $c$. Then, $\lambda: F \rightarrow \mathbb{C}$ mapping $\varphi$ to $c$ is a well-defined linear functional. Since $\varphi_{0} \in F, \lambda$ is surjective. $F^{\prime}$ is the kernel of $\lambda$, and the claim follows. 
Theorem 7. Under the above conditions of Case II, there exists a holomorphic 2-form $\omega_{0}$ on $M$ such that

(1) $\omega_{0}$ is nowhere zero on $M$.

(2) $\omega_{0} \in F$ with $\left\|\omega_{0}\right\|=1$, and

(3) $F=\mathbb{C} \omega_{0} \oplus F^{\prime}$ (orthogonal sum).

The function $\sigma: M \rightarrow \mathbb{R}$ defined by $\sigma(z)=\frac{K(z, \bar{z})}{\omega_{0}(z) \wedge \overline{\omega_{0}(z)}}$ is a biholomorphic invariant. The minimal set of $\sigma$ is exactly $E$. We shall refer to the minimal set of $\sigma$ as the minimal set of the Bergman kernel in this case.

Proof. The existence of $\omega_{0}$ will be verified directly in Section 3 for each case of a rational double point. By Proposition $6, \omega_{0}$ is unique up to a constant multiple $c \omega_{0}$ with $|c|=1$. Hence $\sigma$ is independent of the choice of $\omega_{0}$. Again since $F$ with its inner product is invariant under biholomorphic maps, $\sigma$ is a biholomorphic invariant.

By (2), we may take a complete orthonormal basis $\left\{\omega_{0}, \omega_{1}, \omega_{2}, \cdots\right\}$ of $F$. Then for each $j=1,2,3, \cdots, w_{j} \in F^{\prime}$ by $(3)$ and $\omega_{j}=h_{j} \omega_{0}$ for some holomorphic function $h_{j}$ which vanishes on $E$. Further,

$$
\sigma=\frac{K}{\omega_{0} \wedge \overline{\omega_{0}}}=\frac{\omega_{0} \wedge \overline{\omega_{0}}+\sum \omega_{j} \wedge \overline{\omega_{j}}}{\omega_{0} \wedge \overline{\omega_{0}}}=1+\sum\left|h_{j}\right|^{2} .
$$

Hence $\sigma \geq 1$ on $M$ and $\sigma=1$ on $E$.

To see that $\sigma>1$ on $M \backslash E$, we consider $x \omega_{0}, y \omega_{0}, z \omega_{0}$. As in the previous proof, we get a complete orthonormal basis of $F$ of the form $\left\{\omega_{0}, g_{i} \omega_{0}, \cdots\right\}$, where $g_{i}$ are holomorphic functions on $M$ vanishing simultaneously only on $E$. Hence outside $E$,

$$
\sigma \geq 1+\sum\left|g_{i}\right|^{2}>1
$$

Remark. It is tempting to define $\sigma$ to be $\frac{K}{\omega \wedge \bar{\omega}}$ for arbitrary nowhere vanishing $L^{2}$ holomorphic 2-form $\omega$ on $M$. However, it may then be impossible to locate the exceptional set by the minimal set. For example, if we take $\omega=e^{N x} \omega_{0}$ and fix a point $p \in M$ with $\operatorname{Re}(x)>0$ at $\pi(p)$, then for sufficiently large $N$,

$$
\frac{K}{\omega \wedge \bar{w}}=\frac{K}{w_{0} \wedge \overline{\omega_{0}}} e^{-2 N \operatorname{Re}(x)}\left\{\begin{array}{l}
=1 \text { on } E \\
<1 \text { at } p
\end{array}\right.
$$

\section{Explicit computations}

In case $p_{g}=0,0$ is a rational double point. In this section, we use the explicit resolutions of $A_{n}, n \geq 1, D_{n}, n \geq 4, E_{6}, E_{7}, E_{8}$ (see [M]) to construct the form $\omega_{0}$ in Theorem 7.

Type $\boldsymbol{A}_{n}$.

Let $\widetilde{V}=\left\{(x, y, z) \in \mathbb{C}^{3}: f(x, y, z)=x y-z^{n+1}=0\right\}$.

An explicit resolution $\widetilde{\pi}: \widetilde{M} \rightarrow \widetilde{V}$ can be given in terms of coordinate charts 
and transition functions as follows :

Coordinate charts: $\widetilde{W}_{k}=\mathbb{C}^{2}=\left\{\left(u_{k}, v_{k}\right)\right\}, k=0,1, \cdots, n$.

Transition functions: $\left\{\begin{array}{l}u_{k+1}=\frac{1}{v_{k}} \\ v_{k+1}=u_{k} v_{k}^{2}\end{array} \quad\right.$ or $\quad\left\{\begin{array}{l}u_{k}=u_{k+1}^{2} v_{k+1} \\ v_{k}=\frac{1}{u_{k+1}}\end{array}\right.$

Projection map : $\tilde{\pi}\left(u_{k}, v_{k}\right)=\left(u_{k}^{k+1} v_{k}^{k}, u_{k}^{n-k} v_{k}^{n-k+1}, u_{k} v_{k}\right)$ or

$$
(x, y, z)=\left(u_{0}, u_{0}^{n} v_{0}^{n+1}, u_{0} v_{0}\right)=\cdots=\left(u_{n}^{n+1} v_{n}^{n}, v_{n}, u_{n} v_{n}\right)
$$

Exceptional set : $E=\widetilde{\pi}^{-1}(0)=C_{1} \cup \cdots \cup C_{n}$, where

$$
\begin{aligned}
& C_{k}=\left\{u_{k-1}=0\right\} \cup\left\{v_{k}=0\right\} \\
& \dot{C_{1}} \overline{C_{2}} \cdots-\stackrel{\bullet}{C_{n-1}} \dot{C}_{n}
\end{aligned}
$$

We consider $V=\left\{(x, y, z) \in \mathbb{C}^{3}: x y=z^{n+1}\right.$ and $\left.|x|^{2}+|y|^{2}+|z|^{2}<\varepsilon_{0}\right\}$.

Then $M=\widetilde{\pi}^{-1}(V)$ is given by the coordinate charts:

$$
\begin{array}{r}
W_{k}=\left\{\left(u_{k}, v_{k}\right):\left|u_{k}\right|^{2(k+1)}\left|v_{k}\right|^{2 k}+\left|u_{k}\right|^{2(n-k)}\left|v_{k}\right|^{2(n-k+1)}+\left|u_{k}\right|^{2}\left|v_{k}\right|^{2}<\varepsilon_{0}\right\} \\
k=0,1, \cdots, n
\end{array}
$$

On $M, \quad \varphi_{0}=\frac{d x \wedge d y}{f_{z}}=-\frac{d x \wedge d y}{(n+1) z^{n}}$

$$
=-d u_{k} \wedge d v_{k}, k=0, \cdots, n, \quad \text { by an easy computation. }
$$

Thus, $\varphi_{0}$ is a nowhere vanishing holomorphic 2-form on $M$. Observe that under $\pi: M \rightarrow V, W_{0} \backslash C_{1}$ is mapped biholomorphically onto $V \backslash y$-axis. In particular, $M \backslash W_{0}$ is of measure zero in the obvious sense. Hence, we may compute integrals on $M$ using the $\left(u_{0}, v_{0}\right)$ coordinate on the chart $W_{0}$ alone.

Proposition 8. In the notations for $A_{n}$, let $\varphi_{\alpha \beta}=u_{0}^{\alpha} v_{0}^{\beta} d u_{0} \wedge d v_{0}, \alpha, \beta=$ $0,1,2, \cdots$. Then $\left\{\frac{\varphi_{\alpha \beta}}{\left\|\varphi_{\alpha \beta}\right\|}: \alpha \geq \frac{n}{n+1} \beta\right\}$ is a complete orthonormal basic of $F$.

Proof. The transition functions imply that $u_{k}^{\alpha} v_{k}^{\beta}=u_{k+1}^{2 \alpha-\beta} v_{k+1}^{\alpha}$, hence

$$
u_{0}^{\alpha} v_{0}^{\beta}=\cdots=u_{k}^{(k+1) \alpha-k \beta} v_{k}^{k \alpha-(k-1) \beta}=\cdots=u_{n}^{(n+1) \alpha-n \beta} v_{n}^{n \alpha-(n-1) \beta}
$$

Also, $d u_{k} \wedge d v_{k}=d u_{k+1} \wedge d v_{k+1}$. So, $u_{0}^{\alpha} v_{0}^{\beta} d u_{0} \wedge d v_{0}$ defines a holomorphic 2-form $\varphi_{\alpha \beta}$ on $M$ if and only if $(n+1) \alpha-n \beta \geq 0$. 


$$
\begin{aligned}
& \text { Next, write } u_{0}=r e^{i \theta} \text { and } v_{0}=\rho e^{i \varphi} \text {. Then } \\
& \int_{M} \varphi_{\alpha \beta} \wedge \overline{\varphi_{\alpha^{\prime} \beta^{\prime}}} \\
= & \int_{W_{0}} u_{0}^{\alpha} \overline{u_{0}^{\alpha^{\prime}}} v_{0}^{\beta \overline{\beta_{0}^{\prime}}} d u_{0} \wedge d v_{0} \wedge d \overline{u_{0}} \wedge d \overline{v_{0}} \quad, \text { by the preceding observation } \\
= & 4 \int_{r, \rho \geq 0} r^{\alpha+\alpha^{\prime}+1} \rho^{\beta+\beta^{\prime}+1} d r d \rho \int_{0}^{2 \pi} e^{i\left(\alpha-\alpha^{\prime}\right) \theta} d \theta \\
& \left\{\begin{array}{l}
r^{2}+r^{2 n} \rho^{2(n+1)}+r^{2} \rho^{2}<\varepsilon_{0} \\
=0 \text { if } \alpha \neq \alpha^{\prime} \text { or } \beta \neq \beta^{\prime} \\
<\infty \text { if } \alpha=\alpha^{\prime} \text { and } \beta=\beta^{\prime}
\end{array}\right.
\end{aligned}
$$

Therefore each $\varphi_{\alpha \beta}$ is $L^{2}$ and $\left\{\frac{\varphi_{\alpha \beta}}{\left\|\varphi_{\alpha \beta}\right\|}\right\}$ form an orthonormal system in $F$. Note: the fact that $\varphi_{\alpha \beta}$ is $L^{2}$ also follows from Theorem 3, instead of direct checking as above.

Finally, for any $\varphi \in F, \frac{\varphi}{\varphi_{0}}=h$ is a holomorphic function on $M$. Hence $\varphi=h\left(u_{0}, v_{0}\right) d u_{0} \wedge d v_{0}$ on $W_{0}$. Since $W_{0}$ is a Reinhardt domain, $h\left(u_{0}, v_{0}\right)$ has a convergent power series expansion $h\left(u_{0}, v_{0}\right)=\sum c_{\alpha \beta} u_{0}^{\alpha} v_{0}^{\beta}$ on $W_{0}$. This implies that $\varphi=\sum c_{\alpha \beta} \varphi_{\alpha \beta}$ on $M$, completing the proof.

Observe that $\varphi_{00}=-\varphi_{0}$ while any other $\varphi_{\alpha \beta}$ has $\alpha\left(\geq \frac{n}{n+1} \beta\right) \geq 1$ and so vanishes on $C_{1}$ given by $u_{0}=0$. Also, $\omega_{0}=\frac{\varphi_{0}}{\left\|\varphi_{0}\right\|}$ satisfies all three conditions in Theorem 7.

\section{Type $D_{n}, n \geq 4$ and even.}

Let $\widetilde{V}=\left\{(x, y, z) \in \mathbb{C}^{3}: f(x, y, z)=x^{2} z+y^{2}-z^{n-1}=0\right\}$.

An explicit resolution $\widetilde{\pi}: \widetilde{M} \rightarrow \widetilde{V}$ can be given in terms of coordinate charts and transition functions as follows.

Coordinate charts :

$$
\begin{aligned}
& \qquad \widetilde{W}_{k}=\left\{\left(u_{k}, v_{k}\right): u_{k}^{n-k-3} v_{k}^{n-k-2} \neq 1\right\}, \quad 0 \leq k \leq n-4 \\
& \widetilde{W}_{k}=\left\{\left(u_{k}, v_{k}\right)\right\}, k=n-3, n-2 \\
& \widetilde{W}_{k}=\left\{\left(u_{k}, v_{k}\right): u_{k}^{2} v_{k} \neq-1\right\}, \quad k=n-1, n \\
& \text { Transition functions: }\left\{\begin{array}{l}
u_{k+1}=\frac{1}{v_{k}} \quad \text { or }\left\{\begin{array}{l}
u_{k}=u_{k+1}^{2} v_{k+1} \\
v_{k+1}=u_{k} v_{k}^{2}
\end{array} \quad, 0 \leq k \leq n-3\right. \\
u_{k+1}
\end{array}\right.
\end{aligned}
$$




$$
\begin{aligned}
& \left\{\begin{array} { l } 
{ u _ { n - 1 } = \frac { 1 } { u _ { n - 2 } v _ { n - 2 } } } \\
{ v _ { n - 1 } = u _ { n - 2 } v _ { n - 2 } ^ { 2 } ( 1 - u _ { n - 2 } ) }
\end{array} \quad \text { or } \left\{\begin{array}{l}
u_{n-2}=\frac{1}{1+u_{n-1}^{2} v_{n-1}} \\
v_{n-2}=\frac{1+u_{n-1}^{2} v_{n-1}}{u_{n-1}}
\end{array}\right.\right. \\
& \left\{\begin{array} { l } 
{ u _ { n } = \frac { 1 } { v _ { n - 2 } ( 1 - u _ { n - 2 } ) } } \\
{ v _ { n } = u _ { n - 2 } v _ { n - 2 } ^ { 2 } ( 1 - u _ { n - 2 } ) }
\end{array} \left\{\begin{array}{l}
u_{n-2}=\frac{u_{n}^{2} v_{n}}{1+u_{n}^{2} v_{n}} \\
v_{n-2}=\frac{1+u_{n}^{2} v_{n}}{u_{n}}
\end{array}\right.\right.
\end{aligned}
$$

Projection map : Let $\widetilde{\pi}\left(u_{k}, v_{k}\right)=(x, y, z)$. Then

$$
\begin{aligned}
x & =u_{0}\left(u_{0}^{n-3} v_{0}^{n-2}-1\right)^{\frac{n}{2}-1}\left(u_{0}^{n-3} v_{0}^{n-2}-2\right)=\cdots= \\
& =u_{n-3}^{n-2} v_{n-3}^{n-3}\left(v_{n-3}-1\right)^{\frac{n}{2}-1}\left(v_{n-3}-2\right)=\cdots=\frac{v_{n}^{\frac{n}{2}-1}\left(1-u_{n}^{2} v_{n}\right)}{1+u_{n}^{2} v_{n}} \\
y & =2 u_{0}^{2} v_{0}\left(u_{0}^{n-3} v_{0}^{n-2}-1\right)^{\frac{n}{2}}=\cdots=2 u_{n-3}^{n-1} v_{n-3}^{n-2}\left(v_{n-3}-1\right)^{\frac{n}{2}}=\cdots=\frac{2 u_{n} v_{n}^{\frac{n}{2}}}{1+u_{n}^{2} v_{n}} \\
z & =u_{0}^{2} v_{0}^{2}\left(u_{0}^{n-3} v_{0}^{n-2}-1\right)=\cdots=u_{n-3}^{2} v_{n-3}^{2}\left(v_{n-3}-1\right)=\cdots=v_{n}
\end{aligned}
$$

Exceptional set : $E=\tilde{\pi}^{-1}(0)=C_{1} \cup \cdots \cup C_{k}$, where

$$
\begin{aligned}
& C_{k}=\left\{u_{k-1}=0\right\} \cup\left\{v_{k}=0\right\} \quad 1 \leq k \leq n-2 \\
& C_{n-1}=\left\{v_{n-3}=1\right\} \cup\left\{u_{n-2}=1\right\} \cup\left\{v_{n-1}=0\right\} \\
& C_{n}=\left\{u_{n-2}=0\right\} \cup\left\{v_{n}=0\right\} \\
& C_{n-1} \bullet \\
& \dot{C}_{1} \cdot \overline{C_{2}} \cdots-\dot{C}_{n-2} \dot{C}_{n}
\end{aligned}
$$

For $V=\left\{(x, y, z) \in \mathbb{C}^{3}: x^{2} z+y^{2}-z^{n-1}=0\right.$ and $\left.|x|^{2}+|y|^{2}+|z|^{2}<\varepsilon_{0}\right\}$, $M=\widetilde{\pi}^{-1}(V)$ is given by the coordinate charts:

$$
W_{k}=\left\{\left(u_{k}, v_{k}\right) \in \widetilde{W}_{k}:|x|^{2}+|y|^{2}+|z|^{2}<\varepsilon_{0}\right\}, k=0,1, \cdots, n .
$$

On $M, \varphi_{0}=\frac{d z \wedge d x}{f_{y}}=\frac{d z \wedge d x}{2 y}$

Computation shows that

$$
\begin{aligned}
\varphi_{0} & =d u_{0} \wedge d v_{0}=\cdots=d u_{n-3} \wedge d v_{n-3}=d u_{n-2} \wedge d v_{n-2} \\
& =\frac{d u_{n-1} \wedge d v_{n-1}}{1+u_{n-1}^{2} v_{n-1}}=\frac{d u_{n} \wedge d v_{n}}{1+u_{n}^{2} v_{n}} .
\end{aligned}
$$

Since $1+u_{n-1}^{2} v_{n-1} \neq 0$ on $W_{n-1}$ and $1+u_{n}^{2} v_{n} \neq 0$ on $W_{n}, \varphi_{0}$ is a nowhere zero holomorphic 2-form on $M$. Observe that under $\pi: M \rightarrow V, W_{n-3} \backslash\left(C_{n-1} \cup\right.$ 
$\left.C_{n-2} \cup C_{n-3}\right)$ is mapped holomorphically onto $V \backslash S$, where $S$ consists of curves. Explicitly, the inverse map is given by

$$
\begin{aligned}
& u_{n-3}=\frac{y^{3}\left(z^{n-1}-y^{2}+x z^{\frac{n}{2}}\right)}{2 x\left(4 z^{2(n-1)}-3 y^{2} z^{n-1}+\left(4 z^{n-1}-y^{2}\right) x z^{\frac{n}{2}}\right)} \\
& v_{n-3}=\frac{2}{y^{2}}\left(z^{n-1}+x z^{\frac{n}{2}}\right)
\end{aligned}
$$

for $(x, y, z) \in V$ such that the denominators are nonzero. In particular, $M \backslash W_{n-3}$ is of measure zero and we may compute integrals on $M$ using the $\left(u_{n-3}, v_{n-3}\right)$ coordinates alone.

Proposition 9. $\varphi_{0} \in F$ and $\omega_{0}=\frac{\varphi_{0}}{\left\|\varphi_{0}\right\|}$ satisfies the three conditions in Theorem 7.

Proof. The fact that $\varphi_{0}$ is $L^{2}$ follows from Theorem 3 or by direct checking. It remains only to prove that $\varphi_{0}$, hence $\omega_{0}$, is orthogonal to $F^{\prime}$. Consider any $\omega \in F^{\prime}$. Then $\omega=h \varphi_{0}$ for a holomorphic function on $M$ vanishing on $E$. Using the coordinate chart $W_{n-3}$ and writing $(u, v)=\left(u_{n-3}, v_{n-3}\right)$, we have $\omega=h(u, v) d u \wedge d v$ on $W_{n-3}$. $W_{n-3}$ is given by

$|u|^{2(n-2)}|v|^{2(n-3)}|v-1|^{n-2}|v-2|^{2}+4|u|^{2(n-1)}|v|^{2(n-2)}|v-1|^{n}+|u|^{4}|v|^{4}|v-1|^{2}<\varepsilon_{0}$.

Each plane $\{v=$ constant $\}$ intersects $W_{n-3}$ in a disc with center at the point $(0, v)$ which belongs to $E$. Hence $h(u, v)$ can be written as

$$
h(u, v)=\sum_{\alpha} c_{\alpha}(v) u^{\alpha} \quad \text { with } \quad c_{0}(v)=0
$$

Then $\left(\varphi_{0}, \omega\right)=\int_{M} \varphi_{0} \wedge \bar{\omega}$

$$
\begin{aligned}
& =\int_{W_{n-3}} d u \wedge d v \wedge \sum_{\alpha} \overline{c_{\alpha}(v)} \overline{u^{\alpha}} d \bar{u} \wedge d \bar{v} \\
& =4 \sum_{\alpha}\left(\int \overline{c_{\alpha}(\rho, \varphi)} r^{\alpha} d r d \rho d \varphi \int_{0}^{2 \pi} e^{-i \alpha \theta} d \theta\right), \text { writing } u=r e^{i \theta} \text { and } v=\rho e^{i \varphi} \\
& =0
\end{aligned}
$$

For all the remaining types, we shall show that Proposition 9 also holds for the respective $\varphi_{0}$ 's.

\section{Type $D_{n}, \boldsymbol{n} \geq \mathbf{5}$ and odd.}

Let $\widetilde{V}=\left\{(x, y, z) \in \mathbb{C}^{3}: f(x, y, z)=x^{2}+y^{2} z-z^{n-1}=0\right\}$.

An explicit resolution $\widetilde{\pi}: \widetilde{M} \rightarrow \widetilde{V}$ can be given as follows. The coordinate charts and transition functions are of the same forms as those for the even $n$ type. The projection map is different, namely 


$$
\begin{aligned}
x & =u_{0}^{2} v_{0}\left(u_{0}^{n-3} v_{0}^{n-2}-1\right)^{\frac{n-1}{2}}\left(u_{0}^{n-3} v_{0}^{n-2}-2\right)=\cdots \\
& =u_{n-3}^{n-1} v_{n-3}^{n-2}\left(v_{n-3}-1\right)^{\frac{n-1}{2}}\left(v_{n-3}-2\right)=\cdots=\frac{v_{n}^{\frac{n-1}{2}}\left(1-u_{n}^{2} v_{n}\right)}{1+u_{n}^{2} v_{n}} \\
y & =2 u_{0}\left(u_{0}^{n-3} v_{0}^{n-2}-1\right)^{\frac{n-1}{2}}=\cdots=2 u_{n-3}^{n-2} v_{n-3}^{n-3}\left(v_{n-3}-1\right)^{\frac{n-1}{2}}=\cdots= \\
& =\frac{2 u_{n} v_{n}^{\frac{n-1}{2}}}{1+u_{n}^{2} v_{n}} \\
z & =u_{0}^{2} v_{0}^{2}\left(u_{0}^{n-3} v_{0}^{n-2}-1\right)=\cdots=u_{n-3}^{2} v_{n-3}^{2}\left(v_{n-3}-1\right)=\cdots=v_{n}
\end{aligned}
$$

The exceptional set is also given in the same way as for the even $n$ type.

For $V=\left\{(x, y, z) \in \mathbb{C}^{3}: x^{2}+y^{2} z-z^{n-1}=0\right.$ and $\left.|x|^{2}+|y|^{2}+|z|^{2}<\varepsilon_{0}\right\}$, $M=\widetilde{\pi}^{-1}(V)$ is given by the coordinate charts:

$$
W_{k}=\left\{\left(u_{k}, v_{k}\right) \in \widetilde{W}_{k}:|x|^{2}+|y|^{2}+|z|^{2}<\varepsilon_{0}\right\}, \quad k=0,1, \cdots, n .
$$

On $M, \varphi_{0}=\frac{d y \wedge d z}{f_{x}}=\frac{d z \wedge d z}{2 x}$.

Computation shows that

$$
\begin{aligned}
\varphi_{0} & =d u_{0} \wedge d v_{0}=\cdots=d u_{n-3} \wedge d v_{n-3}=d u_{n-2} \wedge d v_{n-2} \\
& =\frac{d u_{n-1} \wedge d v_{n-1}}{1+u_{n-1}^{2} v_{n-1}}=\frac{d u_{n} \wedge d v_{n}}{1+u_{n}^{2} v_{n}}
\end{aligned}
$$

$\varphi_{0}$ is a nowhere zero holomorphic 2-form on $M$ as before. Again, we may compute integrals on $M$ using the $\left(u_{n-3}, v_{n-3}\right)$ coordinates on $W_{n-3}$ alone. $W_{n-3}$ is given by

$$
\begin{array}{r}
|u|^{2(n-1)}|v|^{2(n-2)}|v-1|^{n-1}|v-2|^{2}+4|u|^{2(n-2)}|v|^{2(n-3)}|v-1|^{n-1}+|u|^{4}|v|^{4}|v-1|^{2} \\
<\varepsilon_{0}
\end{array}
$$

where $(u, v)=\left(u_{n-3}, v_{n-3}\right)$. Since each plane $\left\{v_{n-3}=\right.$ constant $\}$ intersects $W_{n-3}$ in a disc with center belonging to $E$, the proof of Proposition 9 goes through. Hence Proposition 9 also holds for the odd $n$ case.

Type $E_{6}$.

In this case, $\widetilde{V}=\left\{(x, y, z) \in \mathbb{C}^{3}: f(x, y, z)=x^{2}-y^{3}-z^{4}=0\right\}$ and $\widetilde{\pi}: \widetilde{M} \rightarrow \widetilde{V}$ is given as follows.

Coordinate charts: $\widetilde{W}_{k}=\left\{\left(u_{k}, v_{k}\right): u_{k}^{2-k} v_{k}^{3-k} \neq 1\right\}, \quad k=0,1$

$$
\begin{aligned}
& \widetilde{W}_{k}=\left\{\left(u_{k}, v_{k}\right)\right\}, \quad k=2,3 \\
& \left.\widetilde{W}_{4}=\left\{u_{4}, v_{4}\right): u_{4}^{2} v_{4} \neq-1\right\} \\
& \widetilde{W}_{k}=\left\{\left(u_{k}, v_{k}\right): u_{k}^{k-3} v_{k}^{k-4} \neq-1\right\}, \quad k=5,6
\end{aligned}
$$


Transition functions: $\left\{\begin{array}{l}u_{k+1}=\frac{1}{v_{k}} \\ v_{k+1}=u_{k} v_{k}^{2}\end{array} \quad\right.$ or $\left\{\begin{array}{l}u_{k}=u_{k+1}^{2} v_{k+1} \\ v_{k}=\frac{1}{u_{k+1}}\end{array} \quad, \quad k=0,1,2,5\right.$

$$
\begin{aligned}
& \left\{\begin{array} { l } 
{ u _ { 4 } = \frac { 1 } { u _ { 3 } v _ { 3 } } } \\
{ v _ { 4 } = u _ { 3 } v _ { 3 } ^ { 2 } ( 1 - u _ { 3 } ) }
\end{array} \text { or } \left\{\begin{array}{l}
u_{3}=\frac{1}{1+u_{4}^{2} v_{4}} \\
v_{3}=\frac{1+u_{4}^{2} v_{4}}{u_{4}}
\end{array}\right.\right. \\
& \left\{\begin{array} { l } 
{ u _ { 5 } = \frac { 1 } { v _ { 3 } ( 1 - u _ { 3 } ) } } \\
{ v _ { 5 } = u _ { 3 } v _ { 3 } ^ { 2 } ( 1 - u _ { 3 } ) }
\end{array} \text { or } \left\{\begin{array}{l}
u_{3}=\frac{u_{5}^{2} v_{5}}{1+u_{5}^{2} v_{5}} \\
v_{3}=\frac{1+u_{5}^{2} v_{5}}{u_{5}}
\end{array}\right.\right.
\end{aligned}
$$

Projection map:

$$
\begin{aligned}
x & =4 u_{0}^{2}\left(u_{0}^{2} v_{0}^{3}-1\right)^{3}\left(u_{0}^{2} v_{0}^{3}+1\right)=\cdots= \\
& =4 u_{2}^{6} v_{2}^{4}\left(v_{2}-1\right)^{3}\left(v_{2}+1\right)=\cdots=\frac{4 v_{6}^{2}\left(1+2 u_{6}^{3} v_{6}^{2}\right)}{\left(1+u_{6}^{3} v_{6}^{2}\right)^{2}} \\
y & =4 u_{0}^{2} v_{0}\left(u_{0}^{2} v_{0}^{3}-1\right)^{2}=\cdots=4 u_{2}^{4} v_{2}^{3}\left(v_{2}-1\right)^{2}=\cdots=\frac{4 u_{6} v_{6}^{2}}{1+u_{6}^{3} v_{6}^{2}} \\
z & =2 u_{0}\left(u_{0}^{2} v_{0}^{3}-1\right)^{2}=\cdots=2 u_{2}^{3} v_{2}^{2}\left(v_{2}-1\right)^{2}=\cdots=\frac{2 v_{6}}{1+u_{6}^{3} v_{6}^{2}}
\end{aligned}
$$

Exceptional set: $E=C_{1} \cup \cdots \cup C_{6}$, where

$$
\begin{aligned}
& C_{k}=\left\{u_{k-1}=0\right\} \cup\left\{v_{k}=0\right\}, \quad k=0,1,2,3,6 \\
& C_{4}=\left\{v_{2}=1\right\} \cup\left\{u_{3}=1\right\} \cup\left\{v_{4}=0\right\} \\
& C_{5}=\left\{u_{3}=0\right\} \cup\left\{v_{5}=0\right\}
\end{aligned}
$$

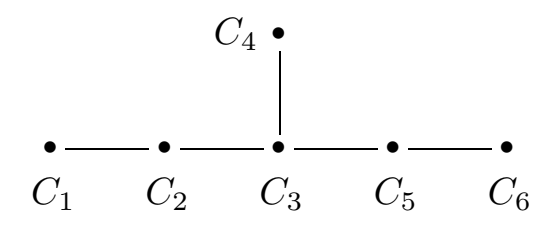

For $V=\left\{(x, y, z) \in \mathbb{C}^{3}: x^{2}-y^{3}-z^{4}=0\right.$ and $\left.|x|^{2}+|y|^{2}+|z|^{2}<\varepsilon_{0}\right\}$, $M=\widetilde{\pi}^{-1}(V)$ is given by the coordinate charts

$$
W_{k}=\left\{\left(u_{k}, v_{k}\right) \in \widetilde{W}_{k}:|x|^{2}+|y|^{2}+|z|^{2}<\varepsilon_{0}\right\}, \quad k=0,1, \cdots, 6
$$

On $M, \varphi_{0}=\frac{d y \wedge d z}{f_{x}}$.

Computation shows that

$$
\begin{aligned}
\varphi_{0} & =d u_{0} \wedge d v_{0}=d u_{1} \wedge d v_{1}=d u_{2} \wedge d v_{2}=d u_{3} \wedge d v_{3} \\
& =\frac{d u_{4} \wedge d v_{4}}{1+u_{4}^{2} v_{4}}=\frac{d u_{5} \wedge d v_{5}}{1+u_{5}^{2} v_{5}}=\frac{d u_{6} \wedge d v_{6}}{1+u_{6}^{3} v_{6}^{2}} .
\end{aligned}
$$

$\varphi_{0}$ is a nowhere zero holomorphic 2-form on $M$ since the denominators are nonzero on the respective coordinate charts. $\pi: M \rightarrow V$ maps $W_{2} \backslash\left(C_{2} \cup C_{3} \cup C_{4}\right)$ 
biholomorphically onto $V \backslash S$, where $S$ consists of curves. Explicitly, the inverse map is given by

$$
u_{2}=\frac{\left(x-z^{2}\right)^{2}}{2 y^{2} z} \quad v_{2}=\frac{\left(x+z^{2}\right)^{2}}{y^{3}}
$$

for $(x, y, z) \in V$ such that the denominators are nonzero. In particular we may compute integrals on $M$ using the $\left(u_{2}, v_{2}\right)$ coordinates alone. $W_{2}$ is given by $16|u|^{12}|v|^{8}|v-1|^{6}|v+1|^{2}+16|u|^{8}|v|^{6}|v-1|^{4}+4|u|^{6}|v|^{4}|v-1|^{4}<\varepsilon_{0}$, where $(u, v)=\left(u_{2}, v_{2}\right)$. Again each plane $\left\{v_{2}=\right.$ constant $\}$ intersects $W_{2}$ in a disc with center belonging to $E$. Hence, Proposition 9 also holds for $E_{6}$.

Type $\boldsymbol{E}_{7}$.

In this case, $\widetilde{V}=\left\{(x, y, z) \in \mathbb{C}^{3}: f(x, y, z)=x^{2}+y^{3}-y z^{3}=0\right\}$ and $\widetilde{\pi}: \widetilde{M} \rightarrow \widetilde{V}$ is given as follows.

Coordinate charts: $\widetilde{W_{k}}, \quad k=0, \cdots, 6$, as for $E_{6}$,

$$
\widetilde{W}_{7}=\left\{\left(u_{7}, v_{7}\right): u_{7}^{4} v_{7}^{3} \neq-1\right\}
$$

Transition functions: As for $E_{6}$, with additional

$$
\left\{\begin{array}{l}
u_{7}=\frac{1}{v_{6}} \\
v_{7}=u_{6} v_{6}^{2}
\end{array}\right.
$$

Projection map:

$$
\begin{aligned}
& x=u_{0}^{3}\left(u_{0}^{2} v_{0}^{3}-1\right)^{5}=\cdots=u_{2}^{9} v_{2}^{6}\left(v_{2}-1\right)^{5}=\cdots=\frac{u_{7} v_{7}^{3}}{\left(1+u_{7}^{4} v_{7}^{3}\right)^{3}} \\
& y=u_{0}^{2}\left(u_{0}^{2} v_{0}^{3}-1\right)^{3}=\cdots=u_{2}^{6} v_{2}^{4}\left(v_{2}-1\right)^{3}=\cdots=\frac{u_{7}^{2} v_{7}^{3}}{\left(1+u_{7}^{4} v_{7}^{3}\right)^{3}} \\
& z=u_{0}^{2} v_{0}\left(u_{0}^{2} v_{0}^{3}-1\right)^{2}=\cdots=u_{2}^{4} v_{2}^{3}\left(v_{2}-1\right)^{2}=\cdots=\frac{v_{7}}{\left(1+u_{7}^{4} v_{7}^{3}\right)^{3}}
\end{aligned}
$$

Exceptional set: $E=C_{1} \cup \cdots \cup C_{7}$, where $C_{1}, \cdots, C_{6}$ are given for $E_{6}$, and $C_{7}=\left\{u_{6}=0\right\} \cup\left\{v_{7}=0\right\}$.

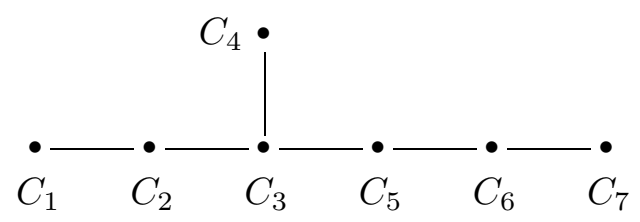

For $V=\left\{(x, y, z) \in \mathbb{C}^{3}: x^{2}+y^{3}-y z^{3}=0\right.$ and $\left.|x|^{2}+|y|^{2}+|z|^{2}<\varepsilon_{0}\right\}$, $M=\widetilde{\pi}^{-1}(V)$ is given by the coordinate charts

$$
W_{k}=\left\{\left(u_{k}, v_{k}\right) \in \widetilde{W}_{k}:|x|^{2}+|y|^{2}+|z|^{2}<\varepsilon_{0}\right\}, \quad k=0,1, \cdots, 7
$$


On $M, \varphi_{0}=\frac{d y \wedge d z}{f_{x}}$.

Computation shows that

$$
\begin{aligned}
\varphi_{0} & =d u_{0} \wedge d v_{0}=\cdots=d u_{2} \wedge d v_{2}=d u_{3} \wedge d v_{3} \\
& =\cdots=\frac{d u_{7} \wedge d v_{7}}{1+u_{7}^{4} v_{7}^{3}}
\end{aligned}
$$

$\varphi_{0}$ is a nowhere zero holomorphic 2-form on $M . \pi: M \rightarrow V$ maps $W_{2} \backslash\left(C_{2} \cup\right.$ $C_{3} \cup C_{4}$ ) biholomorhoically onto $V \backslash S$, where $S$ consists of curves. Explicitly, the inverse map is given by

$$
u_{2}=\frac{y^{3}}{x z^{2}} \quad v_{2}=\frac{z^{3}}{y^{2}}
$$

for $(x, y, z) \in V$ such that the denominators are nonzero. In particular we may compute integrals on $M$ using $\left(u_{2}, v_{2}\right)$ on $W_{2}$. Again, each plane $\left\{v_{2}=\right.$ constant \} intersects $W_{2}$ in a disc with center belonging to $E$. Hence, Proposition 9 holds for $E_{7}$.

\section{Type $E_{8}$.}

In this case, $\widetilde{V}=\left\{(x, y, z) \in \mathbb{C}^{3}: f(x, y, z)=x^{2}-y^{3}+z^{5}=0\right\}$ and $\widetilde{\pi}: \widetilde{M} \rightarrow \widetilde{V}$ is given as follows.

Coordinate charts: $\widetilde{W}_{k}, \quad k=0,1, \cdots, 7$ as for $E_{7}$,

$$
\widetilde{W}_{8}=\left\{\left(u_{8}, v_{8}\right): u_{8}^{5} v_{8}^{4} \neq-1\right\}
$$

Transition functions: As for $E_{7}$ with additional

Projection map:

$$
\left\{\begin{array}{l}
u_{8}=\frac{1}{v_{7}} \\
v_{8}=u_{7} v_{7}^{2}
\end{array}\right.
$$

$$
\begin{aligned}
& x=u_{0}^{5}\left(u_{0}^{2} v_{0}^{3}-1\right)^{8}=\cdots=u_{2}^{15} v_{2}^{10}\left(v_{2}-1\right)^{8}=\cdots=\frac{v_{8}^{3}}{\left(1+u_{8}^{5} v_{8}^{4}\right)^{5}} \\
& y=u_{0}^{4} v_{0}\left(u_{0}^{2} v_{0}^{3}-1\right)^{5}=\cdots=u_{2}^{10} v_{2}^{7}\left(v_{2}-1\right)^{5}=\cdots=\frac{v_{8}^{2}}{\left(1+u_{8}^{5} v_{8}^{4}\right)^{3}} \\
& z=u_{0}^{2}\left(u_{0}^{2} v_{0}^{3}-1\right)^{3}=\cdots=u_{2}^{6} v_{2}^{4}\left(v_{2}-1\right)^{3}=\cdots=\frac{u_{8} v_{8}^{2}}{\left(1+u_{8}^{5} v_{8}^{4}\right)^{2}}
\end{aligned}
$$

Exceptional set: $E=C_{1} \cup \cdots \cup C_{8}$, where $C_{1}, \cdots, C_{7}$ are given as for $E_{7}$, and $C_{8}=\left\{u_{7}=0\right\} \cup\left\{v_{8}=0\right\}$

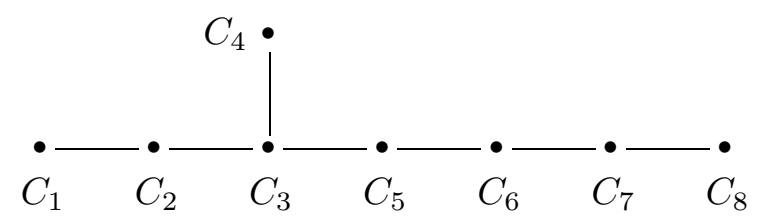


For $V=\left\{(x, y, z) \in \mathbb{C}^{3}: x^{2}-y^{3}+z^{5}=0\right.$ and $\left.|x|^{2}+|y|^{2}+|z|^{2}<\varepsilon_{0}\right\}$, $M=\widetilde{\pi}^{-1}(V)$ is given by the coordinate charts

$$
W_{k}=\left\{\left(u_{K}, v_{k}\right) \in \widetilde{W}_{k}:|x|^{2}+|y|^{2}+|z|^{2}<\varepsilon_{0}\right\}, \quad k=0,1, \cdots, 8 .
$$

On $M, \varphi_{0}=\frac{d y \wedge d z}{f_{x}}$

Computation shows that

$$
\varphi_{0}=-d u_{0} \wedge d v_{0}=\cdots=-d u_{2} \wedge d v_{2}=\cdots=-\frac{d u_{8} \wedge d v_{8}}{1+u_{8}^{5} v_{8}^{4}}
$$

$\varphi_{0}$ is a nowhere zero holomorphic 2-form on $M . \pi: M \rightarrow V$ maps $W_{2} \backslash\left(C_{2} \cup\right.$ $C_{3} \cup C_{4}$ ) biholomorphically onto $V \backslash S$, where $S$ consists of curves. Explicitly, the inverse map is given by

$$
u=\frac{z^{6}}{x y^{2}} \quad v=\frac{y^{3}}{z^{5}},
$$

for $(x, y, z) \in V$ such that the denominators are nonzero. Thus we may compute integrals on $M$ using $\left(u_{2}, v_{2}\right)$ on $W_{2}$. Again, each plane $\left\{v_{2}=\right.$ constant $\}$ intersects $W_{2}$ in a disc with center belonging to $E$. Hence, Proposition 9 holds for $E_{8}$.

Remark. For type $D_{n}$, we can also use the coordinate chart $W_{n-2}$ which intersects each plane $\left\{u_{n-2}=\right.$ constant $\}$ in a disc with center belonging to $E$. For type $E_{n}(n=6,7,8)$, we can also use the coordinate chart $W_{3}$ which intersects each plane $\left\{u_{3}=\right.$ constant $\}$ in a similar disc. The orthogonality of $\varphi_{0}$ to $F^{\prime}$ is not so easy to see in the other coordinate charts for the lack of such an obvious intersection property.

\section{Appendix}

In response to the request of one of the referees, we include this appendix for the convenience of the readers.

Theorem 10. Let $(V, 0)$ be a normal isolated singularity of dimension $n \geq 2$. Suppose that $h$ is a holomorphic function on $V \backslash\{0\}$. Then $h$ is a holomorphic function on $V$.

Proof. Let $\pi: M \longrightarrow V$ be a resolution of singularity with $E=\pi^{-1}(0)$ as an exceptional set. Following Laufer [L2], we consider the sheaf cohomology with compact support and sheaf cohomology with support at infinity. The following sequence is exact.

$$
0 \longrightarrow H_{c}^{0}(M, \mathcal{O}) \longrightarrow H^{0}(M, \mathcal{O}) \longrightarrow H_{\infty}^{0}(M, \mathcal{O}) \longrightarrow H_{c}^{1}(M, \mathcal{O}) \longrightarrow \ldots
$$

Observe that $H_{c}^{1}(M, \mathcal{O})$ is dual to $H^{n-1}\left(M, \Omega^{n}\right)$, where $\Omega^{n}$ is the sheaf of germs of holomorphic $n$-forms, by Serre duality. On the other hand, GrauertRiemenschneider vanishing theorem $[\mathrm{G}-\mathrm{R}]$ asserts that $H^{i}\left(M, \Omega^{n}\right)=0$ for $i \geq 1$. Therefore the restriction mapping $H^{0}(M, \mathcal{O}) \longrightarrow H_{\infty}^{0}(M, \mathcal{O})$ is surjective. It follows that the pull back $\pi^{*} h$ of $h$ on $M \backslash E$ can be extended holomorphically 
on $M$. As a result, $h$ can be extended as a continuous function at the singular point 0 . By normality of $(V, 0), h$ can be extended as an holomorphic function at the singular point 0 .

Theorem 11. Let $(V, 0)$ be a normal isolated singularity of dimension $n \geq 2$. Let $\pi: M \longrightarrow V$ be a resolution of singularity with $E=\pi^{-1}(0)$ as exceptional set. Then any holomorphic $n$-form on $M \backslash E$ can be extended as a meromorphic $n$-form on $M$.

Proof. Let $E=\cup A_{i}, 1 \leq i \leq m$, be an irreducible decomposition of $E$. Without loss of generality, we may assume that $A_{i}$ 's are nonsingular divisors with normal crossing by applying Hironaka Theorem on resolution of singularities. Let $\omega$ be a holomorphic $n$-form on $M \backslash E$. It suffices to prove that $\omega$ has a pole of finite order on each $A_{i}$. Suppose on the contrary that $\omega$ has a pole of infinite order along $A_{i}$. Let $z_{1}$ be the coordinate function which vanishes at 0 . Clearly $\pi^{*}\left(z_{1}\right)$ vanishes along $A_{i}$ with finite order. It follows that for any positive integer $k,\left(\pi^{*}\left(z_{1}\right)\right)^{k} \omega$ cannot be extended holomorphically across $A_{i}$. Therefore $\operatorname{dim} H^{0}\left(M \backslash E, \Omega^{n}\right) / H^{0}\left(M, \Omega^{n}\right)=\infty$, where $\Omega^{n}$ is the sheaf of germs of holomorphic $n$-forms.

On the other hand, we can consider the following exact sequence which relates the sheaf cohomology with compact support and sheaf cohomology with support at infinity

$$
0 \rightarrow H_{c}^{0}\left(M, \Omega^{n}\right) \longrightarrow H^{0}\left(M, \Omega^{n}\right) \rightarrow H_{\infty}^{0}\left(M, \Omega^{n}\right) \rightarrow H_{c}^{1}\left(M, \Omega^{n}\right) \rightarrow H^{1}\left(M, \Omega^{n}\right) .
$$

Obviously $H_{c}^{0}\left(M, \Omega^{n}\right)=0$. Also $H^{1}\left(M, \Omega^{n}\right)=0$ by Grauert-Riemenschneider vanishing theorem. Observe that $H_{\infty}^{0}\left(M, \Omega^{n}\right)=H^{0}\left(M \backslash E, \Omega^{n}\right)$ by Andreotti and Grauert [A-G]. Therefore

$$
\operatorname{dim} H^{0}\left(M \backslash E, \Omega^{n}\right) / H^{0}\left(M, \Omega^{n}\right)=\operatorname{dim} H_{c}^{1}\left(M, \Omega^{n}\right)
$$

$H_{c}^{1}\left(M, \Omega^{n}\right)$ is Serre dual to $H^{n-1}(M, \mathcal{O})$ which is finite dimensional by Andreotti and Grauert $[\mathrm{A}-\mathrm{G}]$. This leads to a contradiction.

\section{References}

[A] M. Artin, On isolated rational singularities of surfaces, Amer. J. Math 88 (1966), 129136.

[A-G] A. Andreotti and H. Grauert, Théorèmes de finitude pour la cohomologie des espaces complexes, Bull. Soc. Math. France 90 (1962), 193-259.

[B1] S. Bergman, The Kernel Function and Conformal Mappings, 2nd Edition, Mathematical Surveys 5, American Mathematical Society, Providence, R.I., 1970.

[B2] Sur les Fonctions Orthogonales de Plusieurs Variables Complexes, Mem. Sci. Math. Paris, no. 106, 1947.

[G-R] H. Grauert and O. Riemenschneider, Verschwindungssätze für analytische Kohomologiegruppen auf komplexen Raumen, Invent. Math. 11 (1970), 263-292.

[K] S. Kobayashi, Geometry of bounded domains, Trans. Amer. Math. Soc. 92 (1959), 267290.

[L1] H. Laufer, Normal Two-Dimensional Singularities, Annals of Mathematics Studies 71, Princeton University Press, Princeton, 1971.

[L2] _ On rational singularities, Amer. J. Math. 94 (1972), 597-608. 
[M] D. R. Morrison, The birational geometry of surfaces with rational double points, Math. Ann. 271 (1985), 415-438.

[Y] S. S.-T.Yau, Two theorems on higher dimensional singularities, Math. Ann. 231 (1977), $55-59$.

Dept. of Mathematics, The Chinese University of Hong Kong, Shatin, Hong Kong. E-mail address: hsluk@math.cuhk.edu.hk

Dept. of Mathematics, Statistics and Computer Science, University of Illinois at ChicAgo, IL 60607-7045.

E-mail address: yau@uic.edu

Dept. of Electronic Engineering, Kunshan Institute of Technology, Tainan, TaiWAN.

E-mail address: yehyu@mail.ksut.edu.tw 